\title{
National trends for open and endovascular repair of aneurysms in Korea: 2004-2013
}

\author{
JIN HYUN JOH, YUN-YOUNG PARK, SUNG-SHIN CHO and HO-CHUL PARK \\ Department of Surgery, Kyung Hee University School of Medicine, Seoul 134-727, Republic of Korea
}

Received July 17, 2015; Accepted September 9, 2016

DOI: 10.3892/etm.2016.3781

\begin{abstract}
The introduction of endovascular aneurysm repair has led to a dramatic decline in open aneurysm repair. The aim of this report was to evaluate Korean national trends for the treatment of aneurysms. A serial, cross-sectional study of time trends of patients who underwent aneurysm repair between 2004 and 2013 was conducted. Data from the Health Insurance Review and Assessment Service were used to evaluate the trends of aneurysm repair in the Korean population and to analyze the trends of open and endovascular aneurysm repair among Medicare beneficiaries. A linear-by-linear association was performed to determine alterations in the rates at which these aneurysm repair techniques were performed. A total of 32,130 patients underwent aneurysm repair between 2004 and 2013. The proportion of patients who underwent open repair decreased from $94.0 \%$ in 2004 to $54.9 \%$ in 2013; whereas the proportion of patients who underwent endovascular repair increased from $6.0 \%$ in 2004 to $45.1 \%$ in 2013. During the study period, the number of patients undergoing endovascular repair of aortic aneurysms significantly increased from 82 to 1,396 (relative risk, 16.17; 95\% confidence interval: 12.94-20.21). Endovascular repair of abdominal aortic aneurysms (AAAs)overtook open repair between 2010 and 2011. The frequency of open aneurysm repair increased 1.2-fold, with an overall downward trend. The prevalence of endovascular repair markedly increased 15.3-fold. These findings indicated that, in Korea, the endovascular repair of AAAs overtook open repair as the most common technique between 2010 and 2011.
\end{abstract}

\section{Introduction}

An aneurysm is defined as an increase in the size of an artery by $>50 \%$ of the normal arterial diameter. According to the

Correspondence to: Professor Jin Hyun Joh, Department of Surgery, Kyung Hee University School of Medicine, 189 Dongnam-ro, Gangdong-gu, Seoul 134-727, Republic of Korea E-mail: jhjoh@khu.ac.kr

Key words: aneurysm, endovascular procedures, open surgery, trend affected segment of the aorta, aneurysmal diseases are divided into thoracic, thoracoabdominal and abdominal aortic aneurysms (AAAs). Worldwide, the prevalence of AAAs may be as high as $8 \%$, depending on the criteria used for diagnosis (1).

Since the introduction of endovascular repair of aortic aneurysms by Parodi et al in 1991 (2), this technique has resulted in lower short-term mortality and shorter hospital stays than open repair. Although endovascular and open repair of AAAs result in similar long-term survival overall, endovascular repair has been shown to increase the long-term survival of younger patients (3). In patients randomized to open or endovascular AAA repair, all-cause perioperative mortality and AAA-related mortality at short- and intermediate-term follow-up was lower in patients who underwent endovascular repair (4).

Due to the attractive outcomes of endovascular repair of aortic aneurysms, the overall use of the endovascular procedure has risen sharply in the last decade. Epidemiology of aortic aneurysm repair in the United States showed that endovascular aneurysm repair (EVAR) increased from 5.2 to $74 \%$ of the total number of AAA repairs from 2000 to 2010, even though the total number of AAAs remained stable at 45,000 cases per year (5). There was a $72 \%$ decline in open repair during the study period. The national trends were similar for the use of open and endovascular repair for thoracic aortic pathologies between 2000 and 2010; the rate of open repair decreased from 2.5 to 2.2 per 100,000 beneficiaries, and the rate of endovascular repair of thoracic aortic aneurysms increased markedly from 0 to 0.8 per 100,000 beneficiaries (6).

There has been little analysis of these national trends in Asian countries. Therefore, the present study aimed to evaluate the national trends for the treatment of aneurysms in Korea.

\section{Materials and methods}

Data collection. Data from the Health Insurance Review and Assessment Service (HIRA) were used to evaluate the trends of aneurysm repair in Korea. Korea has a universal health coverage system, the National Health Insurance, which covers $\sim 98 \%$ of the overall Korean population. Claims data of HIRA are collected from healthcare service providers when they seek reimbursements for healthcare services that the National Health Insurance Corporation covers. These national data are collated from healthcare providers across Korea, and corre- 
spond to the number of claims that are submitted by patients for inclusive healthcare services (7).

The Electronic Data Interchange (EDI) database of HIRA contains operation and management codes. The number of aneurysm repairs was collected from the EDI codes of HIRA. Table I shows the EDI codes for aortic aneurysm repair. Codes for the open repair of aneurysms include O0223, O0224, O2031-O2035, and O2037-O2039; whereas codes O2031, $\mathrm{O} 2032$, and $\mathrm{O} 2033$ are allocated for the open repair of thoracic aortic aneurysms. The open repair of AAAs with/without involvement of the iliac arteries corresponds to codes O0223, O0224 and O2034. Codes O2037 and O2038 are designated for the open repair of unilateral- and bilateral-isolated iliac artery aneurysms, respectively. O2039 includes the open repair of all splanchnic artery aneurysms of the celiac, hepatic, splenic, mesenteric and renal arteries. O2035 indicates the open repair of other arterial aneurysms, with the exception of aneurysms originating from the cardiac and intracranial arteries. Endovascular repair of thoracic aneurysms and AAAs were allocated the codes M6611 and M6612; whereas M6613 denotes the endovascular repair of other arterial aneurysms, with the exception of aneurysms originating from the cardiac and intracranial arteries.

For the evaluation of aneurysm repair for a recent 10 year-period, data from the EDI database of HIRA collected between 2004 and 2013 was used. The total number of aneurysm repairs was calculated for this period and, from this dataset, the trends for open and endovascular repair of aneurysms were analyzed. The present study was approved by the Kyung Hee University Hospital at Gangdong Institutional Review Board (Seoul, Korea

Statistical analysis. A linear-by-linear association was performed to determine the trends of aneurysm repair during the evaluation period. $\mathrm{P}<0.05$ (two-sided) was considered to indicate a statistically significant difference. Data were analyzed using SPSS 19.0 statistical software (SPSS Inc., Chicago, IL, USA).

\section{Results}

Aneurysm repair. A total of 32,130 patients underwent aneurysm repair between 2004 and 2013. Open repair was performed in 23,774 patients, and endovascular repair in 8,356 . The number of patients treated with the respective techniques for each year is detailed in Table II. The proportion of patients who underwent open repair decreased from $94.0 \%$ in 2004 to $54.9 \%$ in 2013 . At the same time, the proportion of patients who underwent endovascular repair increased from $6.0 \%$ in 2004 to $45.1 \%$ in 2013 . The age distribution of patients is shown in Fig. 1. The prevalence of aneurysm repair increased with age: $16 \%$ of patients were in their fifties, $29 \%$ were in their sixties, and $28 \%$ were in their seventies.

Trends in aneurysm repair. The number of patients who underwent aneurysm repair during the study period is presented in Fig. 2. The total number of aneurysm repairs increased significantly from 1,872 in 2004 to 4,032 in 2013 [relative risk (RR) 2.05, 95\% confidence interval (CI)
Table I. EDI codes for the open and endovascular repair of aneurysms.

\begin{tabular}{ll}
\hline EDI codes & \multicolumn{1}{c}{ Type of repair } \\
\hline Open repairs & \\
O0223 & Abdominal aorta, suprarenal \\
O0224 & Abdominal aorta, infrarenal \\
O2031 & Ascending aorta \\
O2032 & Aortic arch \\
O2033 & Descending aorta \\
O2034 & Abdominal aorta involving iliac artery \\
O2035 & Other arteries \\
O2037 & Isolated iliac artery, bilateral \\
O2038 & Isolated iliac artery, unilateral \\
O2039 & Splanchnic artery \\
Endovascular repair & \\
M6611 & Placement of stent graft, aorta \\
M6612 & Placement of stent graft, aorta involving \\
& iliac artery \\
M6613 & Placement of stent graft, other arteries
\end{tabular}

EDI, electronic data interchange.

1.94-2.16). During the study period, the number of patients who underwent open repair significantly increased from 1,759 in 2004 to 2,213 in 2013 (RR 1.20, 95\% CI: 1.12-1.27), with an overall downward trend. However, the number of patients who underwent endovascular repair significantly increased from 113 in 2004 to 1,819 in 2013 (RR 15.29, 95\% CI: $12.65-18.49)$. These trends were all statistically significant $(\mathrm{P}<0.0001)$.

Trends in aortic aneurysm repair. The number of patients who underwent aortic aneurysm repair, excluding those who experienced aneurysms at other sites during the study period, is displayed in Fig. 3. The total number of aortic aneurysm repairs increased significantly from 1,765 in 2004 to 3,565 in 2013 (RR 1.92, 95\% CI: 1.81-2.03). During the study period, the number of open repairs significantly increased from 1,683 in 2004 to 2,169 in 2013 (RR 1.22, 95\% CI: 1.15-1.30), with an overall downward trend. However, the number of endovascular repairs significantly increased from 82 in 2004 to 1,396 in 2013 (RR 16.17, 95\% CI: 12.94-20.21). These trends were all statistically significant $(\mathrm{P}<0.0001)$.

Aortic aneurysm repair per 1,000,000 Medicare Beneficiaries. Population-adjusted frequencies for total, open, and endovascular repair of aortic aneurysms over 10 years are shown in Fig. 4. The total number of aneurysm repairs per 1,000,000 Medicare Beneficiaries significantly increased during the study period, from 39 procedures in 2004 to 70 in 2013 (RR 1.94, 95\% CI, 1.30-2.91; $\mathrm{P}=0.0012$ ). At the same time, the number of open repairs increased from 35 procedures in 2004 to 42 in 2013 (RR 1.20, 95\% CI, 0.77-1.88; $\mathrm{P}=0.4257)$. However, the number of endovascular repairs per 1,000,000 Medicare Beneficiaries significantly 
Table II. Distribution of open and endo repair of aneurysm each year for 10 years (2004-2013).

\begin{tabular}{ccccccccccc}
\hline Year & 2004 & 2005 & 2006 & 2007 & 2008 & 2009 & 2010 & 2011 & 2012 & 2013 \\
\hline Open & 1,759 & 3,444 & 2,232 & 2,424 & 2,895 & 2,078 & 2,091 & 2,288 & 2,350 & 2,213 \\
$(\%)$ & 94.0 & 93.4 & 89.7 & 86.3 & 79.1 & 75.0 & 68.3 & 64.8 & 55.7 & 54.9 \\
Endo & 113 & 242 & 257 & 386 & 763 & 693 & 971 & 1,242 & 1,870 & 1,819 \\
$(\%)$ & 6.0 & 6.6 & 10.3 & 13.7 & 20.9 & 25.0 & 31.7 & 35.2 & 44.3 & 45.1 \\
Total & 1,872 & 3,686 & 2,489 & 2,811 & 3,658 & 2,771 & 3,062 & 3,530 & 4,220 & 4,032 \\
\hline
\end{tabular}

Data are presented as the number of patients who underwent the procedure in the indicated year, with percentages as indicated. Endo, endovascular.

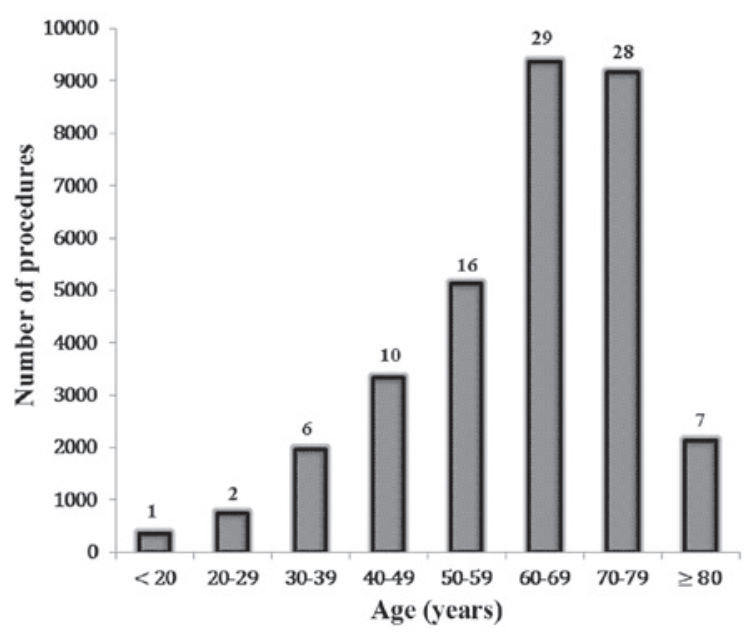

Figure 1. Age distribution for the treatment of aneurysms. The values above the data bars represent the percentage split.between the age ranges.

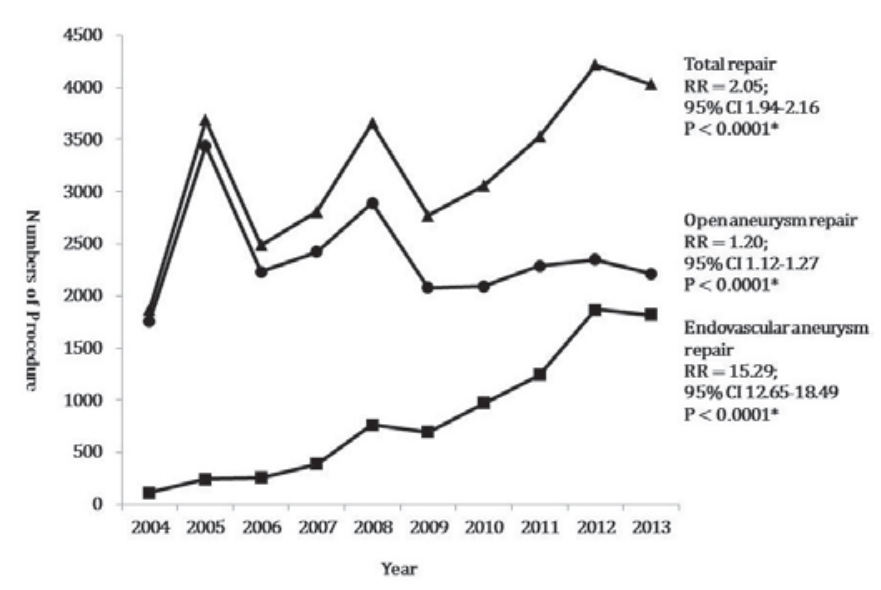

Figure 2. Trends in the total, endovascular, and open repair of aneurysms between 2004 and 2013. *Analyzed by Altman method. RR, risk ratio; CI, confidence interval.

increased, from two procedures in 2004 to 27 in 2013 (RR $13.50,95 \%$ CI, 3.21-56.77; $\mathrm{P}=0.0004)$.

Trends in open aortic aneurysm repair. Changes in the frequency of open repair of aneurysms at each aortic level and in the isolated iliac artery are displayed in Fig. 5. Open repair of the ascending aorta was most commonly performed. The number of ascending aortic aneurysm repairs increased

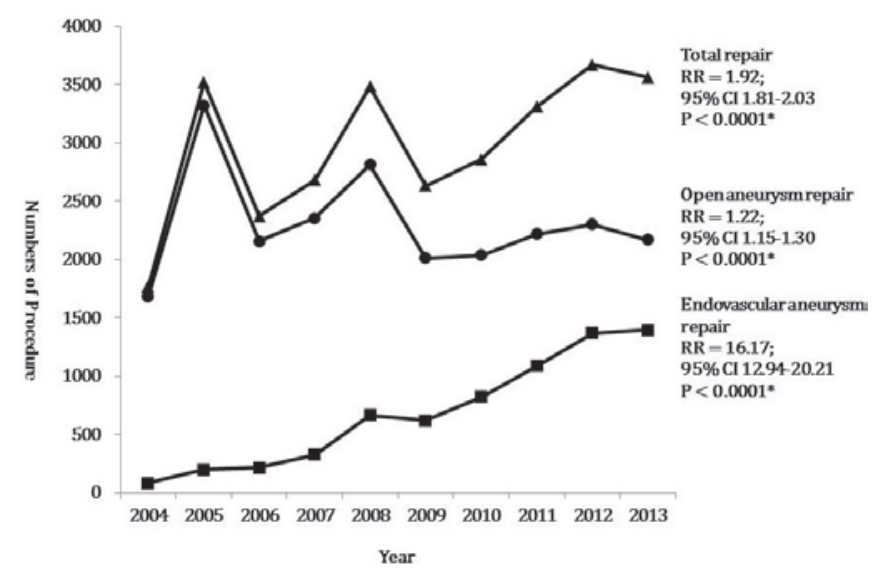

Figure 3. Trends in the total, endovascular, and open repair of aortic aneurysms between 2004 and 2013. "Analyzed by Altman method. RR, risk ratio; $\mathrm{CI}$, confidence interval.

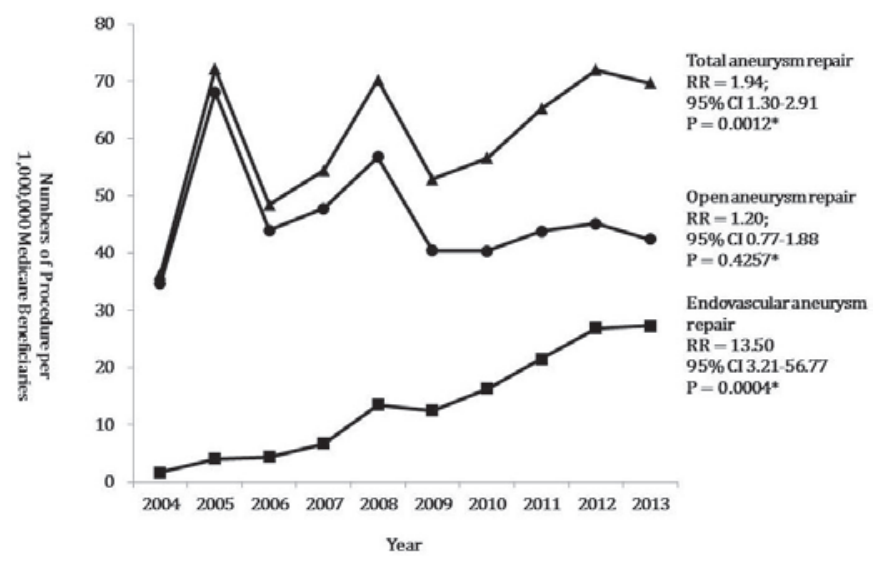

Figure 4. Trends in the total, endovascular and open repair of aortic aneurysms per 1,000,000 Medicare Beneficiaries between 2004 and 2013. *Analyzed by Altman method. RR, risk ratio; CI, confidence interval.

significantly from 601 in 2004 to 876 in 2013 (RR 1.38, 95\% CI: $1.25-1.54 ; \mathrm{P}<0.0001)$. The open repair of AAAs was the second most common procedure, with the number of procedures significantly increasing from 356 in 2004 to 520 in 2014 (RR 1.39, 95\% CI: 1.21-1.59; P<0.0001), with sharp increases in 2005 and 2008. The number of open repairs of the aortic arch performed significantly increased from 230 in 2004 to 399 in 2014 (RR 1.65, 95\% CI: 1.40-1.94; P<0.0001). However, the number of open repairs of descending aortic 


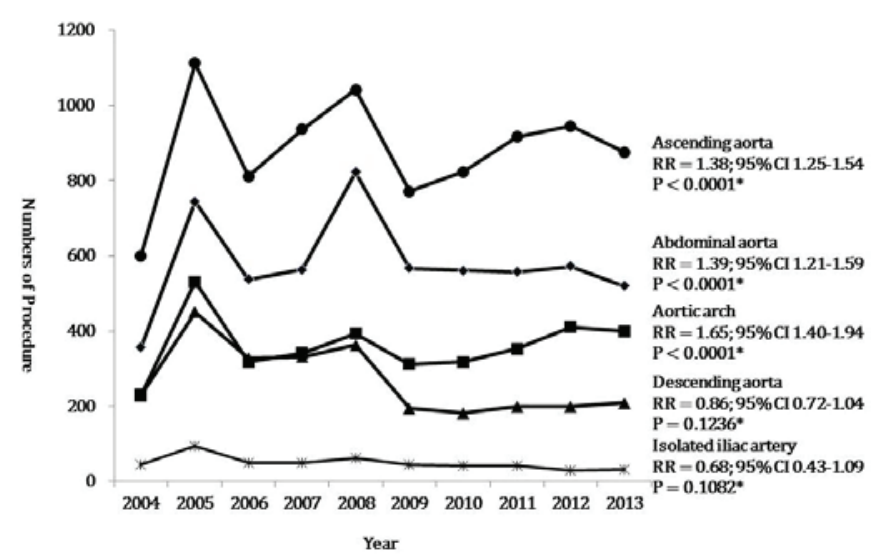

Figure 5. Trends in the open repair of aortic aneurysms at each level between 2004 and 2013. "Analyzed by Altman method. RR, risk ratio; CI, confidence interval.

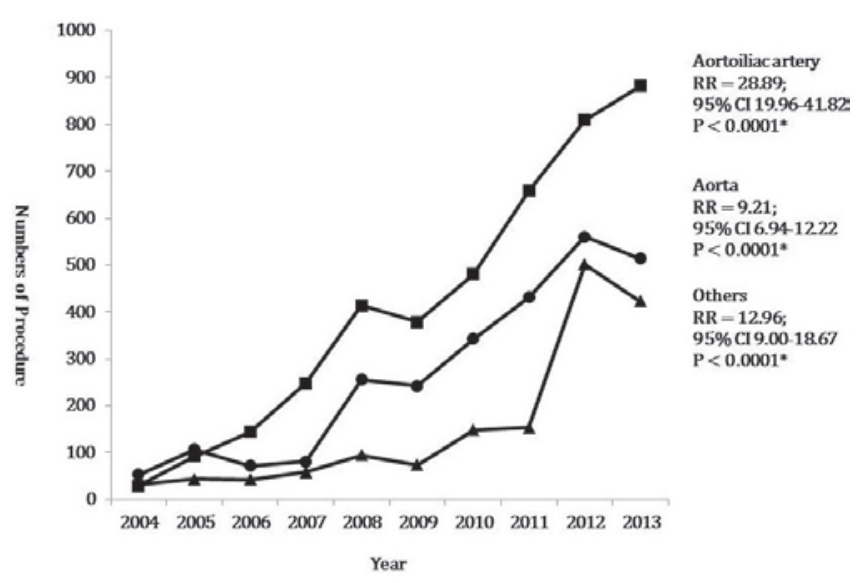

Figure 6. Trends in the endovascular repair of aortoiliac aneurysms, aortic aneurysms, and aneurysms of other arteries between 2004 and 2013 "Analyzed by Altman method. RR, risk ratio; CI, confidence interval.

aneurysms and isolated iliac artery aneurysms performed decreased from 299 to 208 (RR 0.86, 95\% CI: 0.72-1.04; $\mathrm{P}=0.1236)$ and from 43 to 31 (RR 0.68, 95\% CI: 0.43-1.09; $\mathrm{P}=0.1082$ ) between 2004 and 2013, respectively.

Trends in endovascular aneurysm repair. Endovascular repair of aneurysms at all levels dramatically significantly increased from 2004 to 2013 (Fig. 6). The number of endovascular repairs for aortoiliac artery aneurysms significantly increased from 29 in 2004 to 882 in 2013 (RR 28.89 CI: 19.96-41.82). The number of endovascular repairs performed for other aortic aneurysms, including thoracic aortic aneurysms and AAAs using the tube type of stent graft, significantly increased from 53 in 2004 to 514 in 2013 (RR 9.21, 95\% CI: 6.94-12.22). The number of endovascular repairs of other aneurysms, with the exception of aortoiliac and aortic aneurysms, also significantly increased from 31 in 2004 to 423 in 2013 (RR 12.96, 95\% CI: 9.00-18.67). These trends were all statistically significant $(\mathrm{P}<0.0001)$.

Trends in open and endovascular abdominal aortic aneurysm repair. The number of patients who underwent open or endovascular repairs of AAAs is displayed in Fig. 7. The total

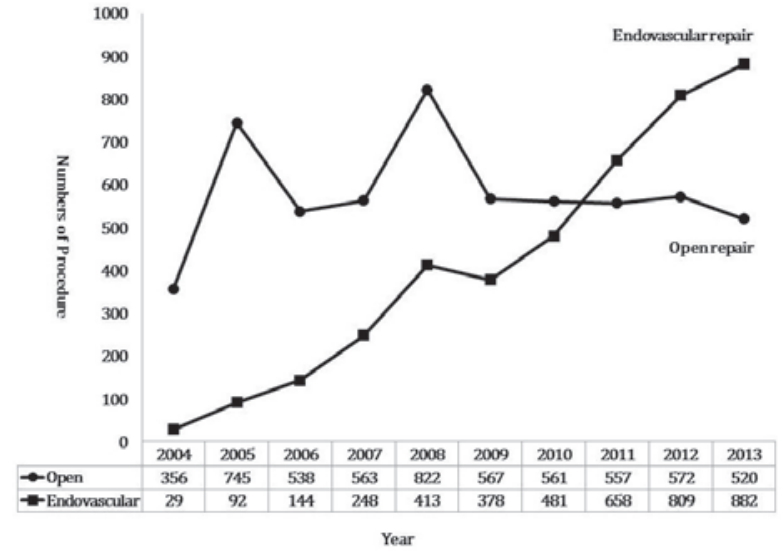

Figure 7. Estimated number of endovascular and open abdominal aortic aneurysm repairs performed in Korea between 2004 and 2013.

number of AAA repairs was 385 in 2004 and 1402 in 2013. With a marked increase in the endovascular repair of AAAs and the downward trend of open AAA repair, endovascular repair overtook open repair as the more popular technique used between 2010 and 2011. Notably, endovascular repair was performed 1.7 times more often than open repair in 2013.

\section{Discussion}

There is a global trend of increasing prevalence of endovascular repair for the treatment of aortic aneurysms. A recent epidemiological study in the US reported that the overall prevalence of endovascular aortic aneurysm repair has risen sharply from $5.2 \%$ in 2000 to $74 \%$ in 2010 (8). In a German study, the rate of treatment by EVAR increased from 24.0 to $40.3 \%$ in males and from 17.3 to $31.0 \%$ in females between 2006 and 2009 (9). In the present study, the rate of endovascular repair of aortic aneurysms increased 16.17 -fold during the study period.

Population-adjusted frequencies of aneurysm repair differ from country to country. According to an epidemiological study in the US, the number of AAA repairs conducted was 14.22 per 100,000 (5). However, in the present study, the total number of aneurysm repairs conducted per 1,000,000 Medicare Beneficiaries was 39 in 2004 and 70 in 2013. Aneurysm repair is performed 2.0-3.6 times more frequently in the US than in Korea.

Several hypotheses have been suggested for the lower rate of aneurysmal repair in Korea. Firstly, the overall incidence of aortic aneurysms is lower in Korea than in Western countries. Ultrasound screening and autopsy series have indicated that the prevalence of AAAs $(\geq 3 \mathrm{~cm})$ is $3-10 \%$ for patients aged $>50$ years old in Western countries (10). In a Veterans Affairs screening study of $>73,000$ patients aged 50-79 years old, the prevalence of AAAs $\geq 3 \mathrm{~cm}$ was $4.6 \%$ (11). In the Asian population, the reported prevalence of AAAs varies. Spark et al (12) suggested that the prevalence of AAAs in the Asian population was lower than that in the Caucasian population. Although a screening study of the general population in Korea demonstrated that the prevalence of AAAs was $4.5 \%$ in a high-risk group (13), another screening study during echocardiograms indicated that $0.2 \%$ of the study population was newly diagnosed with AAAs (14). 
A second explanation for the lower rate of aneurysmal repair in Korea is that there is no nationwide screening program for AAAs. In the US, the Centers for Medicare and Medicaid Services provide free ultrasound screening for AAAs to certain beneficiaries for whom AAA screening may be advantageous, including people who have a family history of AAAs, and men aged 65-75 years old who have smoked $\geq 100$ cigarettes in their lifetime (15). Thirdly, the deployment of medical resources for vascular surgeries in Korea may be different than in Western countries. The Department of Health in the Korean government has focused on the treatment of patients with cancer. The budget for health has been allocated to screen for and treat cancer more so than vascular disease. The deployment of medical resources in Korea may be further evaluated by the health policy authorities.

Despite an overall downward trend in prevalence, the frequency of open repair of aortic aneurysms increased during the study period (1.22-fold). The increased rate of open repair of aortic aneurysms may be associated with the increasing frequency of open repair of ascending aortic aneurysms (RR 1.38, 95\% CI: 1.25-1.54), AAAs (RR 1.39, 95\% CI: 1.21-1.59, P<0.0001), and aortic arch aneurysms (RR 1.65, 95\% CI: 1.40-1.94).

The number of open repairs for descending thoracic aortic aneurysms and isolated iliac artery aneurysms decreased from 299 to 208 (RR 0.86, 95\% CI: 0.72-1.04) and from 43 to 31 (RR 0.68 , 95\% CI: 0.43-1.09) between 2004 and 2013, respectively. This may be associated with the dramatic increase in endovascular repairs of these aneurysms. Endovascular repair of descending thoracic aortic aneurysms is less invasive than open repair and may result in a lower mortality rate, as evidenced by single-center studies or industry-sponsored registries $(16,17)$. Although population-based data from England showed that operative mortality for degenerative descending thoracic aneurysms was similar after either thoracic endovascular aortic/aneurysm repair or open repair (18), endovascular repair is predominantly used for descending thoracic aortic aneurysms.

The iliac branch device (IBD) technique has been introduced as an appealing and effective solution to avoid complications during the repair of aortoiliac aneurysms with extensive iliac involvement, or of isolated iliac artery aneurysms. Perlani et al (19) reported that the estimated patency rate of the internal iliac branch was $91.4 \%$ at 5 years, and the rate of freedom from any re-intervention was $90 \%$ at 1 year and $81.4 \%$ at 5 years. Oderich et al (20) suggested the use of the novel surgeon-modified hypogastric branch stent graft to preserve pelvic perfusion in the circumstance that a commercial IBD is unavailable. Furthermore, Fatima et al (21) published various methods of pelvic revascularization in patients with aortoiliac aneurysms involving one or both common iliac arteries, such as flared iliac stent grafting, the sandwich technique, external-to-internal iliac arterial stenting, double bifurcated stent grafting, or novel hybrid revascularization. In Korea, there are no commercially available IBDs. Therefore, pelvic revascularization is achieved with the surgeon-modified IBD or the novel endovascular or hybrid procedures suggested by Fatima et al (21).

The present study revealed that the endovascular repair of AAAs overtook open repair as the most commonly utilized technique between 2010 and 2011. These results are consistent with a previous epidemiologic study in the US that reported that endovascular repair overtook the open repair of AAAs between 2005 and 2006 (5). This 5-year gap may be related to the late adoption of endovascular treatment for aortic aneurysms and the administrative policies in Korea.

Several limitations of the present study should be acknowledged. As the present study is a retrospective analysis based on an administrative database, there is an intrinsic limit to the number of variables that can be measured. Follow-up data and long-term outcomes were unavailable. In addition, further evaluation is required after more detailed data are collected, such as the number of ruptured and unruptured aortic aneurysms, the prevalence of co-morbidities, the duration of hospital stays, and the rates of mortality according to treatment modality.

In conclusion, a total of 32,130 patients underwent aneurysm repair between 2004 and 2013. The frequency of open repair increased 1.2-fold with an overall downward trend. The prevalence of endovascular repair markedly increased 15.3-fold. Although the frequency of open repair of aortic aneurysms increased at the level of the ascending aorta, aortic arch, and abdominal aorta, the frequency of this technique decreased for descending thoracic aortic aneurysms and isolated iliac artery aneurysms. In Korea, the endovascular repair of AAAs overtook open repair as the most commonly utilized technique between 2010 and 2011.

\section{References}

1. Norman PE and Powell JT: Abdominal aortic aneurysm: The prognosis in women is worse than in men. Circulation 115: 2865-2869, 2007.

2. Parodi JC, Palmaz JC and Barone HD: Transfemoral intraluminal graft implantation for abdominal aortic aneurysms. Ann Vasc Surg 5: 491-499, 1991.

3. Lederle FA, Freischlag JA, Kyriakides TC, Matsumura JS, Padberg FT Jr, Kohler TR, Kougias P, Jean-Claude JM, Cikrit DF and Swanson KM; OVER Veterans Affairs Cooperative Study Group: Long-term comparison of endovascular and open repair of abdominal aortic aneurysm. N Engl J Med 367: 1988-1997, 2012.

4. Dangas G, O'Connor D, Firwana B, Brar S, Ellozy S, Vouyouka A, Arnold M, Kosmas CE, Krishnan P, Wiley J, et al: Open versus endovascular stent graft repair of abdominal aortic aneurysms: A meta-analysis of randomized trials. JACC Cardiovase Interv 5: 1071-1080, 2012.

5. Dua A, Kuy S, Lee CJ, Upchurch GR Jr and Desai SS: Epidemiology of aortic aneurysm repair in the United States from 2000 to 2010. J Vasc Surg 59: 1512-1517, 2014.

6. Jones DW, Goodney PP, Nolan BW, Brooke BS, Fillinger MF, Powell RJ and Stone DH: National trends in utilization, mortality and survival after repair of type B aortic dissection in the medicare population. J Vasc Surg 60: 11-19, 2014.

7. Kim L, Kim JA and Kim S: A guide for the utilization of health insurance review and assessment service national patient samples. Epidemiol Health 36: e2014008, 2014.

8. Dua A, Kuy S, Lee CJ, Upchurch GR Jr and Desai SS: Epidemiology of aortic aneurysm repair in the United States from 2000 to 2010. J Vasc Surg 59: 1512-1517, 2014.

9. Santosa F, Moysidis T, Nowak T, Heilmaier C, Berg C, Luther B and Kröger K: Endovascular abdominal aneurysm repair: Trends in Germany. Vasa 41: 268-274, 2012.

10. Wilmink AB and Quick CR: Epidemiology and potential for prevention of abdominal aortic aneurysm. Br J Surg 85: 155-162, 1998.

11. Lederle FA, Johnson GR, Wilson SE, Chute EP, Littooy FN, Bandyk D, Krupski WC, Barone GW, Acher CW and Ballard DJ: Prevalence and associations of abdominal aortic aneurysm detected through screening. Ann Intern Med 126: 441-449, 1997.

12. Spark JI, Baker JL, Vowden P and Wilkinson D: Epidemiology of abdominal aortic aneurysms in the Asian community. $\mathrm{Br}$ J Surg 88: 382-384, 2001. 
13. Joh JH, Ahn HJ and Park HC: Reference diameters of the abdominal aorta and iliac arteries in the Korean population. Yonsei Med J 54: 48-54, 2013.

14. Oh SH, Chang SA, Jang SY, Park SJ, Choi JO, Lee SC, Park SW, Oh JK and Kim DK: Routine screening for abdominal aortic aneurysm during clinical transthoracic echocardiography in a Korean population. Echocardiography 27: 1182-1187, 2010.

15. Dillavou ED, Muluk SC and Makaroun MS: Improving aneurysm-related outcomes: Nationwide benefits of endovascular repair. J Vasc Surg 43: 446-451; discussion 451-452, 2006.

16. Matsumura JS, Cambria RP, Dake MD, Moore RD, Svensson LG and Snyder S; TX2 Clinical Trial Investigators: Internationa controlled clinical trial of thoracic endovascular aneurysm repair with the Zenith TX2 endovascular graft: 1-year results. J Vasc Surg 47: 247-257; discussion 257, 2008.

17. Makaroun MS, Dillavou ED, Wheatley GH and Cambria RP Gore TAG Investigators: Five-year results of endovascular treatment with the Gore TAG device compared with open repair of thoracic aortic aneurysms. J Vasc Surg 47: 912-918, 2008.
18. von Allmen RS, Anjum A and Powell JT: Outcomes after endovascular or open repair for degenerative descending thoracic aortic aneurysm using linked hospital data. Br J Surg 101: 1244-1251, 2014.

19. Parlani G, Verzini F, De Rango P, Brambilla D, Coscarella C, Ferrer $\mathrm{C}$ and $\mathrm{Cao} P$ : Long-term results of iliac aneurysm repair with iliac branched endograft: A 5-year experience on 100 consecutive cases. Eur J Vasc Endovasc Surg 43: 287-292, 2012.

20. Oderich GS and Ricotta JJ II: Novel surgeon-modified hypogastric branch stent graft to preserve pelvic perfusion. Ann Vasc Surg 24: 278-286, 2010.

21. Fatima J, Correa MP, Mendes BC and Oderich GS: Pelvic revascularization during endovascular aortic aneurysm repair. Perspect Vasc Surg Endovasc Ther 24: 55-62, 2012. 